\title{
Antioxidant and anti-inflammatory activities of pyranoanthocyanins and other polyphenols from staghorn sumac (Rhus hirta L.) in Caco-2 cell models
}

You Peng ${ }^{1 *}$, Hua Zhang ${ }^{2}$, Ronghua Liu $^{2}$, Yoshinori Mine ${ }^{3}$, Jason McCallum ${ }^{4}$, Chris Kirby ${ }^{4}$, Rong Tsao ${ }^{2, *}$

${ }^{1}$ Department of Chemistry and Environment Engineering, Jiujiang University, Jiujiang, China, 332005 ;

${ }^{2}$ Guelph Food Research Centre, Agriculture and Agri-Food Canada, 93 Stone Road West, Guelph, Ontario, Canada N1G 5C9

${ }^{3}$ Department of Food Science, University of Guelph, Guelph, Ontario, Canada N1G 2W1 ${ }^{4}$ Crops and Livestock Research Centre, Agriculture and Agri-Food Canada, 440 University Avenue, Charlottetown, Prince Edward Island, Canada C1A 4N6

* Corresponding authors. Tel.:+86 13755250516; fax:+86 0792-8314448. E-mail address: trihydracid@126.com(You Peng, PhD) ; Tel.: +1 226-217-8108; fax: +1 226-217-8183. E-mail address: rong.cao@agr.gc.ca (Rong Tsao, PhD) 


\begin{abstract}
The antioxidant and anti-inflammatory activities of the novel pyranoanthocyanins and other polyphenols from staghorn sumac (Rhus hirta L.) were examined. The results not only confirmed the previously found antioxidant activity by chemical based assays, but more importantly strong anti-inflammatory activity for the first time in cell-based assays. These unique compounds significantly inhibited the production of pro-inflammatory cytokine IL-8 production stimulated by $\mathrm{H}_{2} \mathrm{O}_{2}$ and TNF- $\alpha$. It was also found that these activities of the crude extract were primarily from the individual polyphenols, particularly the unique pyranoanthocyanins and 7-methoxyanthocyanins. Pyranoanthocyanin 1, in particular, showed a markedly strong anti-inflammatory activity at $1 \mathrm{M}$, followed by pyranoanthocyanin $\mathbf{2}$ and a 7 -methoxyanthocyanin 5. Further mechanism investigation showed that the anti-inflammatory activity was likely mediated through inhibition of $\mathrm{NF}-\kappa \mathrm{B}$ activation via phosphorylation of I $\mathrm{B} \alpha$. Results from the present study warrant further investigation into the in vivo anti-inflammatory effect and health benefits using animal models or human subjects.
\end{abstract}

Keywords: Staghorn sumac; Rhus hirta L.; anti-inflammatory activity; antioxidant activity; IL-8; Caco-2; NF- $\kappa \mathrm{B} ; \mathrm{I} \kappa \mathrm{B} \alpha$ 


\section{Introduction}

Sumac belongs to the genus Rhus of the Anacardiaceae family, which occurs mostly in the tropics and subtropics, but also in the temperate zones, including North America (Gallant, Kemp, \& Lacroix, 1998). These plants are seldom cultivated; some are poisonous, but certain species have been used by indigenous cultures for food and medicinal purposes. This has actually been considered an advantage in commercializing the bioactivity of sumac plants without competing for land uses for food production (Rayne \& Mazza, 2007). The staghorn sumac (Rhus hirta L., synonym: $R$. typhina) is a deciduous shrub to small tree, found in Eastern North America, particularly in Southern Ontario, Canada, although it is now cultivated in China for ecological and ornamental purposes (Rima, Hao, \& Wei, 2011). The fruits of $R$. hirta are used by aboriginals to make beverages or for medicinal purposes (Kossah et al., 2009). The fruits of $R$. hirta have many pharmacological functions including antihaemorrhoidal, antiseptic, blood purifier, diuretic, stomachic, tonic effects, antioxidant and anti-diabetic (McCune \& Johns, 2002; Kossah et al., 2009). Studies also showed that both the fruits of $R$. coriaria and $R$. hirta possess strong antimicrobial and antioxidant activities (McCune \& Johns, 2002; Fazeli et al., 2007; Kossah et al., 2009; Bursal \& Kösal, 2011; Rima et al., 2011). The presence of polyphenols such as gallic acid, hydrolysable tannins, anthocyanins and flavones has been reported in $R$. coriaria (Kosar et al., 2007; Bursal \& Kösal, 2011; Chen \& Chen, 2011). However, only recently, the phytochemical composition and compounds responsible for the antioxidant activities were characterized and reported by our group (Wu et al., 2013; Kirby et al., 2013). Using most recent in vitro methods, we also showed that staghorn sumac had stronger antioxidant activities 
than well-known high-antioxidant fruits such as blueberry and cranberry. Gallic acid, ellagic acid, caffeic acid and its 3-hexose, quercetin and its 3-rhamnoside and uncommon anthocyanins were identified and found to contribute to the antioxidant activities ( $\mathrm{Wu}$ et al., 2013). The pigments were first thought to be anthocyanins as they absorb at similar wavelength, however, most of the aglycones obtained after acid hydrolysis did not match with the known anthocyanidins (Wu et al. 2013). Further studies using mass spectrometry and NMR spectroscopy showed that these unique pigments were not common anthocyanins, but a unique group of pyranoanthocyanins (Kirby et al., 2013).

Meanwhile, antioxidants reduce the health risks related to oxidative stress caused by excess reactive oxygen species such as free radicals. Intestinal tract is one of the most vulnerable organs to free radical attack. Exposure to oxidative stress leads to inflammation. Chronic and acute intestinal inflammations are strongly correlated with intestinal mucosal immune responses. Excessive production of pro-inflammatory molecules from chronic inflammatory process induces perpetuating inflammation in diseases such as inflammatory bowel disease (IBD). Among pro-inflammatory cytokines, TNF- $\alpha$ has been identified to play a critical role in the pathogenesis of IBD in human patients (Neurath et al., 1997; Van Deventer, 1999). Evidence suggests that TNF- $\alpha$ is the major pro-inflammatory cytokine causing the inflammatory cascade through activation of TNF receptor (TNFR)-mediated signaling pathways in IBD, which impairs the mucosal homeostasis and leads to inhibiting the activation of the regulatory system that attenuates pro-inflammatory events. Neutralizing inflammatory responses by anti-TNF- $\alpha$ therapies has been shown to successfully reduce the pathological symptoms and morbidity of 
IBD. However, the anti-TNF- $\alpha$ therapies have significant side-effects, and safe dosage levels for consumption are still controversial (Baumgart \& Sandborn, 2007). Therefore, novel therapeutic approaches need to be developed for IBD treatment.

Polyphenols are strong antioxidants and anti-inflammatory agents. Some dietary polyphenols-based interventions have been found with therapeutic efficacy to attenuate the inflammation and oxidative stress (Kim et al., 2011; Zhang et al., 2013; Lee et al., 2014; Florinda et al., 2014; Antonella et al., 2014). These interventions have a limited toxic effect compared with the pharmaceutical approaches used to treat the patients (Murakami, 2014; Lina et al., 2012). Continued effort in developing new dietary polyphenols-based strategy targeting TNF- $\alpha$-induced inflammatory and oxidative stress signaling events is a valid and necessary approach. The aim of this study is therefore to further investigate the anti-inflammatory and antioxidant activities of the identified polyphenols of $R$. hirta, including the unique pyranoanthocyanins, using an in vitro model of inflammation in Caco-2 epithelial cell lines, and to evaluate the polyphenol-mediated effects on TNF- $\alpha$-induced activations of NFאB and MAPK pathways in Caco-2 cells. Information obtained in the present study will provide insight into developing $R$. hirta or its polyphenols based novel strategies for treating and managing chronic inflammatory diseases such as IBD, and other tract inflammation.

\section{Materials and Methods}

\subsection{Chemicals and reagents}

The following antibodies were used in this study: rabbit monoclonal anti-phospho-SAPRK/JNK, 
anti-SAPRK/JNK, and mouse monoclonal anti-phospho-IкB $\alpha$, and $\beta$-actin (Cell Signaling Technology, Danvers, MA, USA); and HRP-conjugated anti-mouse and -rabbit IgG (Promega, Madison, WI, USA). The foetal bovine serum (FBS) Hyclone, Dulbecco's modified Eagle's medium (DMEM/F12) (Invitrogen Life Technologies Inc., Burlington, ON, Canada). Mature staghorn sumac (R. hirta) fruits were harvested in October, 2013 (Guelph, ON, Canada). The clusters of red hairy fruits were collected with gentle rubbing by hand. The collected fruits (110 g) were blended in $1100 \mathrm{~mL}$ of $0.1 \%$ trifluoroacetic acid (v/v) in $80 \%$ ethanol (v/v). Extractions were carried out on a rotary shaker (Scientific Industries Inc., Tewksbury, MA, USA) overnight (ca. $16 \mathrm{~h} ; 400 \mathrm{rpm})$ at room temperature $\left(22^{\circ} \mathrm{C}\right) . \quad$ After centrifugation, the remaining supernatant was reduced to water via rotary evaporation (Heidolph Instruments, Schwabach, Germany), and then lyophilized (LabConco Corporation, Kansas City, MO, USA), generating a crude extract powder, from which the unique anthocyanins (compounds 1-6) of $R$. hirta were prepared in-house as described in Kirby et al. (2013) through flash chromatography and semi-preparative HPLC, and confirmed of the structures by matching with those previously identified peaks. Compounds $\mathbf{1}$ and $\mathbf{2}$ are pyranoanthocyanins: $\mathbf{1}$, 7-O-methyldelphinidin-3-O-(2"galloyl)-galactoside- 3"'-O-glucoside-4-vinylcatechol (Sumadin A-3-O-(2"galloyl)-galactoside-3"'-O-glucoside); 2, Sumadin B-3-O-(2"galloyl)-galactoside-3"'-O-glucoside. Compounds 3-6 are also unique anthocyanins found in staghorn sumac, with 3-5 to be 7-methoxylanthocyanins: 3, 7-O-methyl-cyanidin-3-O-(2"galloyl)-galactoside, 4, 7-O-methylcyanidin-3-O-galactoside), 5 7-O-methyl-delphinidin-3-O-(2"galloyl)-galactoside; 6, cyanidin-3- $O$-(2"galloyl)-galactoside; 
compounds 7-10 are other phenolics we previously identified in staghorn sumac and they were purchased from Sigma-Aldrich (St. Louis, MO, USA): 7, gallic acid; 8, quercetin; 9, ellagic acid; 10, peonidin chloride. The crude ethanolic extract was used as sample $\mathbf{1 1}$ in the present study. Structures of staghorn sumac polyphenols are shown in Figure 1.

\subsection{Cell Culture and Treatment}

The human colorectal adenocarcinoma-derived intestinal epithelial cell (IEC) line Caco-2 was purchased from American Type Culture Collection (ATCC) (Manassas, VA, USA). Caco-2 cells were grown in $5 \mathrm{~mL}$ of Dulbecco's Modified Eagle Medium: Nutrient Mixture F-12 (DMEM/F12) (Invitrogen Life Technologies Inc, Burlington, ON, Canada) supplemented with 20\% FBS (HyClone) and $50 \mathrm{U} / \mathrm{mL}$ penicillin-streptomycin (Invitrogen Life Technologies Inc.,).

For treatment with polyphenols, cells between passages 20-40 were seeded at a density of $2 \times 10^{5}$ cells/well in 48-well plates (Corning, Life Science, Tewksbury, MA, USA) and grown for 5-7 days with fresh medium replacements every 2-3 days. Confluent cell monolayers were rinsed with Hank's buffered salt solution (HBSS) and treated with different concentrations of polyphenols $\left(10^{-4} \sim 10^{-8} \mathrm{M}\right)$ which were dissolved in culture medium at doses and times indicated. For in vitro inflammation experiments, Caco-2 cells were stimulated with recombinant human TNF- $\alpha$ ( $2 \mathrm{ng} / \mathrm{mL}$; Invitrogen) to induce inflammation. For in vitro antioxidant experiments, Caco-2 cells were stimulated with $\mathrm{H}_{2} \mathrm{O}_{2}(1 \mathrm{mM}$; Sigma-Aldrich, Oakville, ON, Canada) to induce oxidative stress. The cell viability was examined by water-soluble tetrazolium-1 (WST-1, Roche Diagnostics, Laval, QC, Canada) assay as described by Hegenauer et al. (1977). After the removal of supernatant for the IL- 8 assays, $200 \mu \mathrm{L}$ WST- 1 containing medium was added into 
each well of the plate and incubated for $10-30 \mathrm{~min}$ at $37{ }^{\circ} \mathrm{C}$ until colour development. Supernatants were transferred into a 96-well microtitre plate for measurement at $450 \mathrm{~nm}$ in a plate reader (BioRad model 550, Bio-Rad Laboratories, Hercules, CA, USA ).

\subsection{Preparation of stock and working solutions for cell experiments}

The stock solution of polyphenols were prepared at $50 \mathrm{mM}$ in dimethyl sulfoxide (DMSO) and stored at $-30{ }^{\circ} \mathrm{C}$ (except for 11 which was at $25 \mathrm{mg} / \mathrm{mL}$ ). Serial dilutions of polyphenols from $10^{-8} \mathrm{M}$ to $10^{-4} \mathrm{M}$ were prepared by adding 5\% FBS DMEM/ F12 medium at a final volume of 0.5

$\mathrm{mL}$ (the concentration range of 11 was $0.01-100 \mu \mathrm{g} / \mathrm{mL})$. WST-1 was diluted by 1:30 (v/v) in $5 \%$ FBS DMEM/ F12 medium as working solution and stored at $-30{ }^{\circ} \mathrm{C}$. The recombinant human TNF- $\alpha(1 \mathrm{~mL}, 100 \mathrm{ng} / \mu \mathrm{L})$ was diluted with $1000 \mu \mathrm{L}$ 5\% FBS DMEM/ F12 medium or HBSS for the assays. $\quad \mathrm{A} \mathrm{H}_{2} \mathrm{O}_{2}$ working solution at $1 \mathrm{mM}$ was prepared by adding $5.13 \mu \mathrm{L}$ of $30 \% \mathrm{H}_{2} \mathrm{O}_{2}$ into 45 mL 5\% FBS DMEM/ F12 medium.

\subsection{Evaluation of anti-inflammatory activity}

Staghorn sumac polyphenols were supplemented in 5\% FBS containing DMEM/F12 medium before adding TNF- $\alpha$. For each experiment, there were positive (TNF- $\alpha$ treated) and negative controls (without TNF- $\alpha$ treatment). The entire medium was first removed by siphoning from wells where cell monolayers are confluent. Then the wells with cell monolayers were washed with $500 \mu \mathrm{L}$ HBSS and added the working solutions of compounds 1-11 into separate wells for incubation. After $2 \mathrm{~h}$, the cells were stimulated with $10 \mu \mathrm{L}$ TNF- $\alpha$ working solution (except for the negative control) and incubated for $4 \mathrm{~h}$. Cell viability was examined by WST-1 assay. The cell culture supernatants were collected and stored in $-80{ }^{\circ} \mathrm{C}$ freezer before measuring 
IL-8 secretion by Enzyme-Linked Immunosorbent Assay (ELISA) kit (eBioscience, Inc., San Diego, CA, USA). .

\subsection{Evaluation of antioxidant activity}

Staghorn sumac polyphenols were supplemented in 5\% FBS containing DMEM/F12 medium also before stimulated with $\mathrm{H}_{2} \mathrm{O}_{2}$. For each experiment, a positive $\left(\mathrm{H}_{2} \mathrm{O}_{2}\right.$ treated) and negative control (without $\mathrm{H}_{2} \mathrm{O}_{2}$ treatment) were included. The wells with cell monolayers were washed with $500 \mu \mathrm{L}$ HBSS and added the working solutions of polyphenols 1-11. After incubation for $2 \mathrm{~h}$, the entire medium was removed from the wells and the cells were washed by $500 \mu \mathrm{L}$ HBSS. The cells were stimulated with $500 \mu \mathrm{L} \mathrm{H}_{2} \mathrm{O}_{2}$ working solution and incubated for 6 h. The negative control was added with $500 \mu \mathrm{L}$ medium. Cell viability was examined by WST-1 assay. The cell culture supernatants were collected and stored in $-80^{\circ} \mathrm{C}$ freezer for measuring IL-8 secretion using the same ELISA kit.

\subsection{Analysis of Cytokine IL-8 by ELISA}

The IL-8 concentration in the above collected cell supernatants was analyzed using the same IL-8 ELISA kit as stated above. Mouse anti-human IL-8 antibodies (Cat.554716, BD Bioscience, San Diego, CA, USA) were coated at $0.1 \mu \mathrm{L}$ per well in $100 \mu \mathrm{L}$ of $0.1 \mathrm{M}$ sodium phosphate $(\mathrm{pH}$ 9.0) buffer in a Costar® 96-well microtiter plate (Corning, NY, USA), and incubated overnight at $4{ }^{\circ} \mathrm{C}$. The wells were washed 4 times with $300 \mu \mathrm{L}$ of phosphate buffered saline (PBS), pH 7.0, containing $0.05 \%$ Tween-20 (PBST) and then shaken with $200 \mu \mathrm{L} 1 \%(\mathrm{w} / \mathrm{v})$ bovine serum albumin (BSA) in PBS for $1 \mathrm{~h}$ at $37^{\circ} \mathrm{C}$ for blocking. The plate was washed 4 times between each

of the following steps. IL-8 standard (Cat. 558465, BD Bioscience, Mountain View, CA, USA) 
was diluted with $1 \%$ BSA in PBS to prepare working solutions ranging from 32.25 to 1000 pg/mL. The cell supernatant samples and IL-8 standard working solution were added into the plate which was shaken for $2 \mathrm{~h}$ at $37^{\circ} \mathrm{C}$ after blocking. The biotinylated mouse anti-human IL- 8 antibodies $100 \mu \mathrm{L}$ (Cat.554718, BD Bioscience, Mississauga, ON, Canada) were added into the plate at a dilution rate of 1:2000 (v/v) with $1 \%$ BSA in PBST for $1 \mathrm{~h}$ incubation at $37^{\circ} \mathrm{C}$. The avidin-horseradish peroxidase conjugate (HRP) (Cat.554058, BD Bioscience Mississauga, ON, Canada) $(100 \mu \mathrm{L})$ was placed into each well at a dilution rate of 1:2000 (v/v) with $1 \%$ BSA in PBST and incubated for $0.5 \mathrm{~h}$ at $37^{\circ} \mathrm{C}$. After the final step of washing, the colour development was completed by adding $50 \mu \mathrm{L} /$ well of 3,3',5,5'-tetramethylbenzidine (TMB; Sigma-Aldrich) and incubated for 5-15 min at room temperature in the dark. The colour development reaction was stopped by $25 \mu \mathrm{L} /$ well $0.5 \mathrm{M} \mathrm{H}_{2} \mathrm{SO}_{4}$. Finally the absorbance was read at $450 \mathrm{~nm}$. IL-8 concentrations in the samples were extrapolated from the IL-8 standard calibration curve.

\subsection{Immunoblotting analysis}

\subsubsection{Cell samples preparation}

For treatment with polyphenols, Caco-2 cells between passages 20-40 were seeded at a density of $2 \times 10^{5}$ cells/well in 24-well plates (Corning) and let grow for 5-7 d with fresh medium replacement every 2-3 d. Confluent cell monolayers were rinsed with HBSS and treated with $1 \mathrm{~mL} 10^{-4} \mathrm{M}$ of polyphenol compounds $\mathbf{2 , 3 , 5}$ and $\mathbf{6}$ dissolved in culture medium. After $2 \mathrm{~h}$, the cells were stimulated with $20 \mu \mathrm{L}$ TNF- $\alpha$ working solution and incubated for additional $1 \mathrm{~h}$. The non-treated (negative) control cells were not treated with TNF- $\alpha$. The cell viability was examined by WST-1 assay. The cells were washed twice by cold PBS and then stored directly 
under $-80^{\circ} \mathrm{C}$ for further immunoblotting analysis.

\subsubsection{Immunoblotting analysis}

The cells were lysed in $100 \mu \mathrm{L}$ ice-cold radioimmunoprecipitation assay (RIPA) buffer (Thermo Fisher, Rockford, IL, USA) containing Halt ${ }^{\mathrm{TM}}$ Protease and Phosphatase Inhibitor Cocktail (Thermo Fisher). Cell lysates were sonicated for $5 \mathrm{~min}$ at room temperature and centrifuged at $175 \mathrm{r} / \mathrm{s}$ for $25 \mathrm{~min}$ at $4{ }^{\circ} \mathrm{C}$, and protein concentration was measured by DC Protein Assay (Bio-Rad, Mississauga, ON, Canada). Samples (30-50 $\mu \mathrm{g}$ of protein) were separated by SDS-PAGE (10\%) and transferred to a nitrocellulose membrane (Bio-Rad). Membranes were blocked using $5 \mathrm{~mL} 5 \%$ non-fat milk powder in Tris-buffered saline (TBS), and incubated with 5 $\mathrm{mL}$ primary antibody at the dilution rate of $1: 2000$ or $1: 1000(\mathrm{v} / \mathrm{v})$ overnight at $4{ }^{\circ} \mathrm{C}$. Detection was carried out using $5 \mathrm{~mL}$ of HRP-conjugated anti-mouse or anti-rabbit IgG (Promega, Madison, MA, USA) at the dilution rate of 1:10,000 (v/v) and ECL Western Blotting Detection Reagent

(GE Healthcare, Mississauga, ON, Canada). Densitometry was performed using Image J software (Image Processing and Analysis in Java, National Institutes of Health, http://rsbweb.nih.gov/ij/).

\subsection{Statistical analysis}

Data are expressed as means \pm SEM. Statistical analyses were performed with GraphPad Prism version 5.0 (GraphPad) using one-way ANOVA followed by Tukey's multiple-comparison test. Differences were considered significant when $p<0.05$.

\section{Results and Discussion}




\subsection{Anti-inflammatory effect of staghorn sumac polyphenols}

The anti-inflammatory activities of the crude ethanolic extract and the major polyphenols of staghorn sumac were assessed using an in vitro model of inflammation in Caco- 2 cells. Cells were treated with TNF- $\alpha$ to induce cellular inflammatory responses; and secretion of the chemokine IL-8 was measured as an indicator of inflammation (Figure 2). As expected, TNF- $\alpha$ significantly increased IL-8 secretion (positive control) to $\geq 6$ fold of the level of the untreated cells (negative control). Treatment with sumac polyphenols including the extract significantly reduced TNF- $\alpha$-induced production of the pro-inflammatory chemokine IL-8 in a dose-dependent manner. This effect was observed when cells were pre-treated with the polyphenols and the crude extract for $2 \mathrm{~h}$ prior to TNF- $\alpha$ addition (Figure 2), indicating that the ethanol extract and the individual polyphenols of $R$. hirta could act to prevent inflammation.

The anti-inflammatory experiments indicated that compound $\mathbf{1}$ (or sumadin A glycoside), a unique pyranoanthocyanin of staghorn sumac, showed the strongest activity with significant reduction of TNF- $\alpha$-induced IL-8 at as low as $1 \mu \mathrm{M}(p<0.05)$, followed by another pyranoanthocyanin, compound $\mathbf{2}$ (or sumadin B glycoside) and a rare 7-methoxylated anthocyanin $\mathbf{5}$ and the crude extract $\mathbf{1 1}$ which showed significant anti-inflammatory activity at 10 $\mu \mathrm{M}$ (or $10 \mu \mathrm{g} / \mathrm{mL}$ for 11 ) (Table 1 ). Two other 7 -methoxy anthocyanins $\mathbf{3}, \mathbf{4}$, a cyanidin glycoside 6 and ellagic acid (9) showed significant activity at $100 \mu \mathrm{M}$ (Figure 2). Gallic acid (7), quercetin (8), and peonidin (10) did not show significant effect on the TNF- $\alpha$-induced IL-8 secretion at the concentrations used in this experiment $(\leq 100 \mu \mathrm{M})$ (Figure 2). No compounds showed any anti-inflammatory activity below $0.1 \mu \mathrm{M}$ or $1 \mu \mathrm{g} / \mathrm{mL}$ for the extract. Data obtained 
suggest that the anti-inflammatory activity of staghorn sumac crude extract (sample 11), as assessed by inhibition of TNF- $\alpha$-induced IL-8 secretion in Caco- 2 cells, may be from its polyphenolic constituents especially the naturally occurring pyranoanthocyanins and 7-methoxylated anthocyanins.

\subsection{Antioxidant activity of staghorn sumac polyphenols}

Similar to the above-mentioned anti-inflammatory activity, Caco-2 cells were used to evaluate the antioxidant activity. Instead of using TNF- $\alpha$ as a pro-inflammatory agent, $\mathrm{H}_{2} \mathrm{O}_{2}$ was used in this experiment to induce oxidative stress. Oxidative stresses such as those from $\mathrm{H}_{2} \mathrm{O}_{2}$ are well known to enhance IL-8 production in Caco-2 cells (Ivison et al., 2010), therefore IL-8 was again used as a biomarker for antioxidant activity. The expression level of IL-8 was drastically elevated to over 6 fold of that in untreated Caco-2 cells (negative control) after exogenous $\mathrm{H}_{2} \mathrm{O}_{2}$ stimulation (Figure 3). When Caco-2 cells were pretreated with staghorn sumac polyphenols before exposure to $\mathrm{H}_{2} \mathrm{O}_{2}$, the cellular oxidative stress was considerably decreased as seen in significantly reduced production of the chemokine IL-8 (Figures 3). The antioxidant activity of the sumac extract or its polyphenols as shown in reduced IL-8 production was also generally dose-dependent, and similar to the anti-inflammatory effect, the antioxidant activity is considered as a protectant against oxidative stress.

The antioxidant experiment indicated that pyranoanthocyanin 1, gallic acid (7) and quercetin (8) were strong antioxidants as seen in significant reduction of $\mathrm{H}_{2} \mathrm{O}_{2}$-induced IL-8 production at $10 \mu \mathrm{M}$ level (Figure 3). Other pyranoanthocyanin or anthocyanins 2, 5 and $\mathbf{6}$, ellagic acid (9) and the extract 11 showed significant antioxidant activity at $100 \mu \mathrm{M}$ (or $\mu \mathrm{g} / \mathrm{mL}$ for the extract) 
level, whereas compounds $\mathbf{3}, \mathbf{4}$ and $\mathbf{1 0}$ did not significantly affect the IL-8 production (Figure 3). It should be noted that quercetin $(8)$ at higher concentration $(\geq 100 \mu \mathrm{M})$ enhanced IL-8 production, thus may be a prooxidant under such conditions (Figure 3D)(Table 2). Flavonoids may have a dual role of being an antioxidant and a prooxidant. Most flavonoids, especially when at high concentrations, can play a significant role in promoting oxidation. Schmalhausen et al. (2007) reported that at concentrations higher than $200 \mu \mathrm{M}$, quercetin can promote the formation of hydroxyl radical and production of $\mathrm{H}_{2} \mathrm{O}_{2}$, especially in the presence of ferrous ion, whereas it protected oxidation in the absence of a metal ion. All polyphenols at below $1 \mu \mathrm{M}$ showed no antioxidant activity in this assay, and no compounds other than quercetin at any tested concentrations showed prooxidant activity. These results suggest that polyphenols particularly the pyranoanthocyanins in the staghorn sumac extract are important contributors to the antioxidant activity as measured by $\mathrm{H}_{2} \mathrm{O}_{2}$-induced IL-8 production in Caco- 2 cells.

The antioxidant activity of sumac polyphenols did not necessarily correspond to the anti-inflammatory effect (Figures 2 and 3). Among all sumac polyphenols, the two pyranoanthocyanins $\mathbf{1}$ and $\mathbf{2}$, distinctive to staghorn sumac, were most interesting in that they were both strong antioxidants and anti-inflammatory agents (Figures $2 \mathrm{~A}$ and $3 \mathrm{~A}$ ). The unique addition of the pyran ring ( $\mathrm{D}$ ring) to the $\mathrm{A}$ and $\mathrm{C}$ rings of the anthocyanin structure, and further extension by a phenolic group on the pyran ring (Figure 1), have made these two compounds highly conjugated, capable of neutralizing free radicals such as those generated from $\mathrm{H}_{2} \mathrm{O}_{2}$. While many antioxidants also possess anti-inflammatory activities, the mechanism of the latter by the unique pyranoanthocyanins $\mathbf{1}$ and $\mathbf{2}$ are not known. Compounds $\mathbf{3}$ and $\mathbf{4}$ are 
7-methoxylated anthocyanins that are rarely found in commonly consumed foods such as fruits and vegetables. While they showed significant anti-inflammatory effect against TNF- $\alpha$ (Figure 2B), these two compounds did not show antioxidant activity against $\mathrm{H}_{2} \mathrm{O}_{2}$ (Figure 3B). Compound $\mathbf{5}$ and $\mathbf{6}$ showed both significant antioxidant and anti-inflammatory activities $(\mathrm{P}<0.05)$ (Figures $2 \mathrm{C}$ and $3 \mathrm{C}$ ), with $\mathbf{5}$ to be a stronger anti-inflammatory agent. A common structural feature of compounds 1-5 is the 7-methoxy group on A ring, but only $\mathbf{1}$ and $\mathbf{5}$ have a galloyl feature on $\mathrm{B}$ ring $\left(\mathrm{R}^{\prime}=\mathrm{OH}\right)$. The galloyl feature of $\mathrm{B}$ ring contributes to a possible mesomeric equilibrium of the phenoxyl radical of the compound, in which the semiquinone structure has been reported to be the most stable mesomeric structures (Taubert et al., 2003). These two structural features may be important for both anti-inflammatory and antioxidant activities (Figures 2A, 2C, 3A and 3C). Compounds $\mathbf{2 , 3}$ and $\mathbf{4}$ shared a common catechol structure in the B ring, which may be more important for the anti-inflammatory than the antioxidant activity as only $\mathbf{2}$ showed significant antioxidant activity among the three compounds (Figure 3). Although structure-activity relationship is outside of the scope of this study, precise contributions of the different structural features of unique anthocyanins such as the pyranoanthocyanins of sumac will help better understand the mechanisms of action and find use of such plant resources for food and health. Compared to other anthocyanins, the aglycone peonidin had neither antioxidant nor anti-inflammatory activities at concentrations tested in this study.

\subsection{Possible mechanism of the anti-inflammatory activity of pyranoanthocyanins and}

\section{anthocyanins}


Secretion of the pro-inflammatory chemokine IL-8 is known to be regulated by the transcription factor NF-אB (Lee et al., 2013; Ham et al., 2015; Zhang et al., 2015). As such, it is important to understand the molecular mechanism of sumac polyphenols involved in inflammatory signaling, i.e. whether the inhibition of the TNF- $\alpha$-induced IL- 8 production by the staghorn sumac polyphenols as found in the present study was mediated through inhibition of NF- $\kappa \mathrm{B}$ activation, i.e. phosphorylation of I $\kappa \mathrm{B} \alpha . \quad$ To further validate the hypothesis, Caco- 2 cells were pre-treated with selected sumac polyphenols with anti-inflammatory activity, and then exposed to TNF- $\alpha$ before analyzed for phosphorylated IкB $\alpha(p-I \kappa B \alpha) . \quad$ As shown in Figure 4, $\mathrm{p}-\mathrm{I} \kappa \mathrm{B} \alpha$ was significantly elevated by the treatment of TNF- $\alpha$ after $1 \mathrm{~h}$ incubation, however, in cells pre-treated with sumac pyranoanthocyanins or anthocyanins $(\mathbf{2}, \mathbf{3}$ and $\mathbf{6})$, the expression of TNF- $\alpha$-induced $\mathrm{p}-\mathrm{I} \kappa \mathrm{B} \alpha$ was significantly reduced $(\mathrm{P}<0.05)$. This result suggests that polyphenols, particularly the pyranoanthocyanins and anthocyanins of the staghorn sumac exert their strong anti-inflammatory effects by modulating TNF- $\alpha$-induced signaling events, i.e. inhibition of the phosphorylation of $\mathrm{I} \kappa \mathrm{B} \alpha$ early in the pathway. This is the first report of the anti-inflammatory effect of the pyranoanthocyanins, and the first that showed such activity may be mediated through the inhibition of TNF- $\alpha$-induced $N F-\kappa B$ activation via phosphorylation of $\mathrm{I} \kappa \mathrm{B} \alpha$.

The strong antioxidant and anti-inflammatory effects of sumac pyranoanthocyanins and anthocyanins as observed in the present and other studies (Azevedo et al., 2014) naturally bring up the question of their bioaccessibility and bioavailability in vivo. While many studies have confirmed the serum uptake, albeit relatively low, of anthocyanins in animal and human 
experiments, specific data on the bioavailability of pyranoanthocyanins have not been reported in the literature. Pyranoanthocyanins such as Vitisin A derivatives in aged wines have been studied and found to be substantially more bioaccessible, i.e. more stable to gastrointestinal conditions in vitro, which could lead to enhanced bioavailability and bio-effectiveness in vivo as suggested by the authors (McDougall et al., 2005). Cell culture study on wine anthocyanins and vitisins showed similar protective effects against TNF-a-induced MCP-1 secretion in primary human endothelial cells (Garcia-Alonso et al., 2004), suggesting once absorbed, pyranoanthocyanins may exert similar biological activities. However, absorption in the body is only one of the factors affecting the ultimate bio-effectiveness. Better bioaccessibility (McDougall et al., 2005) and the modulatory effect of the epithelial cells of the gut also suggest that these compounds can potentially amelioratechronic inflammation and mucosal injury along the gastrointestinal tract without being absorbed into the blood stream. The lack of specific information on the bioaccessibility and bioavailability of the pyranoanthocyanins do however warrant further studies in the direction. A recent review on anthocyanins also called for new research on the absorption, bioavailability and putative effects on human health of anthocyanins including pyranoanthocyanins (Santos-Buelga, Mateus, \& De Freitas, 2014).

In summary, the present study not only confirmed the previously found strong antioxidant activity of the staghorn sumac extract with a new cell-based antioxidant assay measuring the inhibition of IL-8 production as stimulated by hydrogen peroxide, but also showed that the cell-based antioxidant activity of the sumac extract were primarily from the polyphenols, particularly the unique pyranoanthocyanins and anthocyanins found only in the staghorn sumac 
$R$. hirta. Moreover, these unique anthocyanins also showed strong anti-inflammatory activities as was found in significantly inhibited IL-8 production induced by the pro-inflammatory factor TNF- $\alpha$ in Caco-2 cells pretreated with the extract or individual compounds. Pyranoanthocyanin 1 in particular showed significantly strong anti-inflammation at $1 \mu \mathrm{M}$, followed by pyranoanthocyanin 2 and anthocyanin 5. Further investigation into the possible mechanism of the anti-inflammatory effects showed that staghorn sumac polyphenols, particularly the pyranoanthocyanins and anthocyanins, exert the anti-inflammatory activity via inhibition of TNF- $\alpha$-induced IL-8 production which is mediated through inhibition of NF-KB activation via phosphorylation of $I \kappa B \alpha$. Results from the present study warrant further investigation into the in vivo anti-inflammatory effect and health benefits using animal models or human subjects. Further exploration of the polyphenol-rich fruits of staghorn sumac and development of functional foods or beverages based on these anti-inflammatory components, particularly the pyranoanthocyanins, are under way.

\section{Acknowledgements}

This project is supported by the A-Base research (RBPI 1004) of Agriculture \& Agri-Food Canada. The authors also acknowledge the support from the National Science Foundation of China (No. 81160412), and Special Fund for Young Teacher Development Plan (visiting scholars) in Universities of Jiangxi Province, China. We are grateful to Dr. Yaning Shi for her technical assistance. 


\section{References}

Antonella, G., Imperatrice, C., Angela, C., Isabella, D., Vito, L., Flavia, P., Fiorenza, M.(2014). Antioxidant activity induced by main polyphenols present in edible artichoke heads: influence of in vitro gastro-intestinal digestion. Journal of Functional Foods, 10, 456-464.

Azevedo, J., Oliveira, J., Cruz, L., Teixeira, N., Brás, N.F., De Freitas, V., Mateus, N. (2014) Antioxidant features of red wine pyranoanthocyanins: experimental and theoretical approaches. Journal of Agricultural and Food Chemistry, 62(29),7002-7009.

Baumgart, D. C., \&Sandborn, W. J. (2007). Gastroenterology 2: Inflammatory bowel disease: clinical aspects and established and evolving therapies. Lancet. 369, 1641-1657.

Bursal, E., \&Kösal, E. (2011). Evaluation of reducing power and radical scavenging activities of water and ethanol extracts from sumac (Rhuscoriaria L.). Food Research International, 44, 2217-2221.

Chen, G., \& Chen, H. (2011). Extraction and deglycosylation of flavonoids from sumac fruits using steam explosion. Food Chemistry, 126, 1934-1938.

Fazeli, M. R., Amin, G., Attari, M. M. A., Ashtiani, H., Jamalifar, H., \&Samadi, N. (2007). Antimicrobial activities of Iranian sumac and avishaneshirazi (Zataria multiflora) against some food-borne bacteria.Food Control, 18, 646-649.

Florinda, F., Federica, C., Autilia, C., Tiziana, G., Donatella, A., Marisa, D. M., Massimo, Z., Raffaele, C., Filomena, N.(20140. Polyphenol composition and antioxidant activity of different grass pea (Lathyrussativus), lentils (Lens culinaris), and chickpea (Cicer arietinum) ecotypes of the Campania region (Southern Italy).Journal of Functional Foods, 7, 551-557. 
Gallant, J. B., Kemp, J. R., \&Lacroix, C. R. (1998).Floral development of dioecious Staghorn Sumac, Rhushirta (Anacardiaceae).International Journal of Plant Sciences, 159, 539-549.

Garcia-Alonso, M., Rimbach, G., Rivas-Gonzalo, J. C.,Pascual-Teresa de, S. (2004). Antioxidant and cellular activities of anthocyanins and their corresponding vitisins A-studies in platelets, monocytes, and human endothelial cells. Journal of Agricultural and Food Chemistry,52, $3378-3384$.

Ham, Y. M., Ko, Y. J., Song, S. M., Kim, J., Kim, K. N., Yun, J. H., Cho, J. H., Ahn, G., Yoon, W. J. Y.(2015). Anti-inflammatory effect of litsenolide B2 isolated from Litsea japonica fruit via suppressing NF-kB and MAPK pathways in LPS-induced RAW264.7 cells. Journal of Functional Foods, 13, 80-88.

Hegenauer J., Ripley L. \&Nace, G. (1977).Staining acid phosphoproteins (phosvitin) in electrophoretic gels.Analytical Biochemistry, 78, 308-311.

Ivison, S. M., Wang, C., Himmel, M. E., Sheridan, J., Delano, J., Mayer, M. L., Yao, Y., Kifayet, A., \&Steiner, T. S.(2010). Oxidative stress enhances IL-8 and inhibits CCL20 production from intestinal epithelial cells in response to bacterial flagellin. American Journal of Physiology - Gastrointestinal and Liver Physiology,299, G733-741.

Kim,J. S., Ahn, J., Lee, S. J., Moon, B. K., Ha, T. Y.,\&Kim, S. (2011). Phytochemicals and antioxidant activity of fruits and leaves of paprika(Capsicum annuumL.var. special) cultivated in Korea. Journal of Food Science, 76, 193-198.

Kirby, C. W., Wu, T., Tsao, R., \&McCallum, J. L. (2013).Isolation and structural characterization of unusual pyranoanthocyanins and related anthocyanins from Staghorn sumac (Rhustyphina L.) 
via UPLC-ESI-MS, (1)H, (13)C, and 2D NMR spectroscopy. Phytochemistry, 94, 284-293.

Kosar, M., Bozan, B., Temelli, F., \& Baser, K. H. C. (2007). Antioxidant activity and phenolic composition of sumac (Rhuscoriaria L.) extracts. Food Chemistry, 103, 952-959.

Kossah, R., Nsabimana, C., Zhao, J., Chen, H., Tian, F., Zhang, H., Chen W. (2009).Comparative study on the chemical composition of Syrian Sumac (Rhuscoriaria L.) and Chinese Sumac (Rhustyphina L.) fruits.Pakistan Journal of Nutrition, 8, 1570-1574.

Lee, H. Seon Lee., Bilehal, D., Lee, G. S., Ryu, D. S., Kim, H. K., Suk, D. H., Lee, D. S. (2013). Anti-inflammatory effect of the hexane fraction from Orostachysjaponicus in RAW 264.7 cells by suppression of NF-אB and PI3K-Akt signaling. Journal of Functional Foods, 5(3), 1217-1225.

Lee, W. C., Jao, H. Y., Hsu, J. D., Lee, Y. R., Wu, M. J., Kao, Y. L., Lee, H. J.(2014). Apple polyphenols reduce inflammation response of the kidneys in unilateral ureteral obstruction rats. Journal of Functional Foods, 11, 1-11.

Lina, B.A.R., Reus, A.A.,Hasselwander, O., Bui, Q.,Tenning, P.P.(2012). Safety evaluation of Evesse $^{\mathrm{TM}}$ EPC, an apple polyphenol extract rich in flavan-3-ols.Food and Chemical Toxicology, $50(8), 2845-2853$.

McCune, L. M., \& Johns, T. (2002).Antioxidant activity in medicinal plants associated with the symptoms of diabetes mellitus used by the indigenous peoples of the North American boreal forest. Journal of Ethnopharmacology, 82, 197-205.

McDougall, G. J., Fyffe, S., Dobson, P., Stewart, D. (2005).Anthocyanins from red wine - Their stability under simulated gastrointestinal digestion. Phytochemistry, 66, 2540-2548. 
Murakami, A.(2014). Dose-dependent functionality and toxicity of green tea polyphenolsin experimental rodents.Archives of Biochemistry and Biophysics, 557,3-10.

Neurath, M. F., Fuss, I. J., Pasparakis, M., Alexopoulou, L., Haralambous, S., MeyerzumBuschenfelde, K.H., Stronber, W., \&Kollias, G. (1997). Predominant pathogenic role of tumor necrosis factor in experimental colitis in mice.European Journal of Immunology, 27, $1743-50$.

Rayne, S., \&Mazza, G. (2007). Biological activities of extracts from Sumac (Rhus spp.): A review. Plant Foods for Human Nutrition (Formerly QualitasPlantarum), 62, 165-175.

Rima, K., Hao, Z., \& Wei, C. (2011). Antimicrobial and antioxidant activities of Chinese sumac (Rhustyphina L.) fruit extract. Food Control, 22, 128-132.

Santos-Buelga, C., Mateus, N., De Freitas, V. (2014). Anthocyanins.Plant pigments and beyond. Journal of Agricultural and Food Chemistry, 62(29), 6879-6884.

Schmalhausen, E. V., Zhlobek, E. B., Shalova, I. N., Firuzi, O.,Saso, L.,\&Muronetz, V. I. (2007). Antioxidant and prooxidant effects of quercetin on glyceraldehyde-3-phosphate dehydrogenase.Food and Chemical Toxicology, 45, 1988-1993.

Taubert, D., Breitenbach, T., Lazar, A., Censarek, P., Harlfinger, S., Berkels, R., Klaus, W., Roesen, R. (2003). Reaction rate constants of superoxide scavenging by plant antioxidants. Free Radic. Biol. Med. 35, 1599-1607.

VanDeventer, S. J. (1999). Anti-TNF antibody treatment of Crohn's disease.Annals of the Rheumatic Diseases, 58, 1114-20.

Wu, T., McCallum, J. L., Wang, S., Liu, R., Zhu, H., \&Tsao, R. (2013). Evaluation of 22 
Antioxidant Properties and Chemical Characterization of Staghorn Sumac Fruit (RhushirtaL.).Food Chemistry, 138, 1333-1340.

Zhang, H., Jennifer K.-N., Tomohiro K., Yuzuru E., Yoshinori M.(2015). $\gamma$-Glutamyl cysteine and $\gamma$-glutamyl valine inhibit TNF- $\alpha$ signaling in intestinal epithelial cells and reduce inflammation in a mouse model of colitis via allosteric activation of the calcium-sensing receptor. BiochimicaetBiophysicaActa, 1852, 792-804.

Zhang, Y. R.,Tang,T.,Huang, Y.,Li, Y.,Xie, L.,Zhan, X.,Tang, Z. H.,\&Tang, J. T. (2013). Study on anti-oxidation and anti-inflammatory activity ofCapsicum annuum leaves polyphenols. Science and Technology of Food Industry, 8, 346-349. 


\section{Figure Captions}

Figure 1. Structures of polyphenols isolated from the fruit body of staghorn sumac (Rhus hirta L.). Pyranoanthocyanins (1 and 2): 1, Sumadin A -3-O-(2"galloyl)-galactoside-3"'-O-glucoside; 2, Sumadin B -3-O-(2"galloyl)-galactoside-3"'-O-glucoside. 7-O-methoxy anthocyanins (3-5): 3, 7-O-methyl-cyanidin-3-O-(2"galloyl)-galactoside, 4, 7- $O$-methyl-cyanidin-3- $O$-galactoside), 5 7-O-methyl-delphinidin-3-O-(2"galloyl)-galactoside; 6, cyanidin-3-O-(2"galloyl)-galactoside; 7 , gallic acid; 8 , quercetin; 9 , ellagic acid; 10, peonidin.

Figure 2. Anti-inflammatory activity of pyranoanthocyanins, 7-methoxy anthocyanins and other polyphenols isolated from staghorn sumac and its $80 \%$ ethanol extract. Caco-2 cells were pretreated with compounds for $2 \mathrm{~h}$ at indicated concentrations and stimulated with TNF- $\alpha$ for $4 \mathrm{~h}$. IL-8 secretion was measured by ELISA. Data are expressed as means \pm SEM of three independent experiments. $* \mathrm{P}<0.05$, $* * \mathrm{P}<0.01$, and $* * * \mathrm{P}<0.001$ relative to the positive control ( $\mathrm{PC}, \mathrm{TNF}-\alpha)$ cells. *a $\mathrm{P}<0.05$, **a $\mathrm{P}<0.01$, ***a $\mathrm{P}<0.001$ relative to the negative control (NC, normal) cells. \#: the concentration of 11 is $\mathrm{mg} / \mathrm{L}$.

Figure 3. Antioxidant activity of pyranoanthocyanins, 7-methoxy anthocyanins and other polyphenols isolated from staghorn sumac and its $80 \%$ ethanol extract. Caco-2 cells were pretreated with compounds for $2 \mathrm{~h}$ at indicated concentrations and stimulated with $\mathrm{H}_{2} \mathrm{O}_{2}$ for $6 \mathrm{~h}$. IL-8 secretion was measured by ELISA. Data are expressed as means \pm SEM of three independent experiments. $* \mathrm{P}<0.05$, $* * \mathrm{P}<0.01$, and $* * * \mathrm{P}<0.001$ relative to the positive control $\left(\mathrm{PC}, \mathrm{H}_{2} \mathrm{O}_{2}\right)$ cells. *a $\mathrm{P}<0.05$, **a $\mathrm{P}<0.01$, ***a $\mathrm{P}<0.001$ relative to the negative control (NC, normal) cells. \#: the concentration of 11 is $\mathrm{mg} / \mathrm{L}$.

Figure 4. Inhibition of TNF- $\alpha$-induced phosphorylation of $\mathrm{p}-\mathrm{IkB} \alpha$ by selected staghorn sumac pyranoanthocyanins and anthocyanins. Protein expression was analyzed by Western blotting. Data are expressed as means \pm SEM of three independent. Cells were pre-treated with $100 \mu \mathrm{M}$ compounds 2, 3, 5 and $\mathbf{6}$ for $1 \mathrm{~h}$ followed by stimulation with TNF- $\alpha$ for indicated time points. $* \mathrm{a} \quad \mathrm{P}<0.05, * * \mathrm{a} \quad \mathrm{P}<0.01 * * * \mathrm{a} \quad \mathrm{P}<0.001$ relative to the control (normal) cells in the same group. $* \mathrm{~b} \mathrm{P}<0.05, * * \mathrm{~b} \mathrm{P}<0.01 * * * \mathrm{~b} \mathrm{P}<0.001$ relative to the positive control (TNF- $\alpha$ ) cells in the same group. 
Figure 1

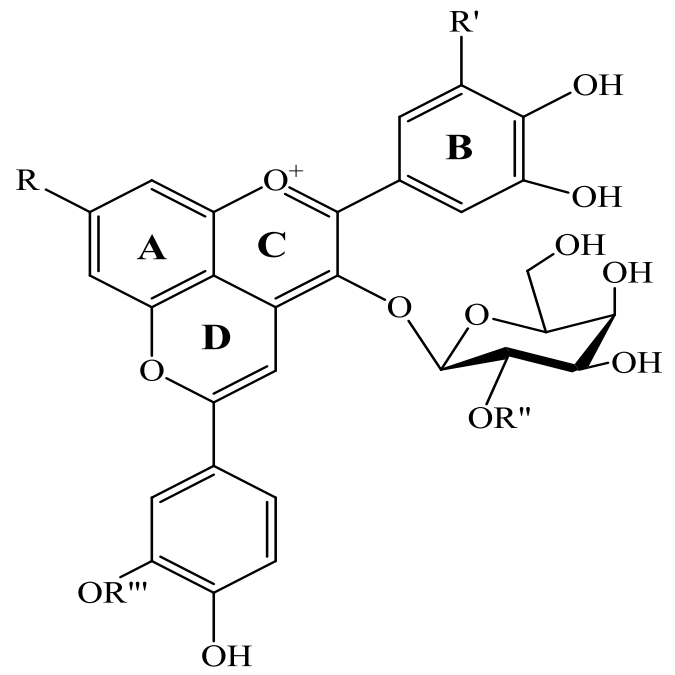

$1 \mathrm{R}=\mathrm{OCH}_{3}, \mathrm{R}^{\prime}=\mathrm{OH}, \mathrm{R} "=$ galloyl, $\mathrm{R} "=$ glucosyl $2 \mathrm{R}=\mathrm{OCH}_{3}, \mathrm{R}^{\prime}=\mathrm{H}, \mathrm{R} "=$ galloyl, R"'=glucosyl

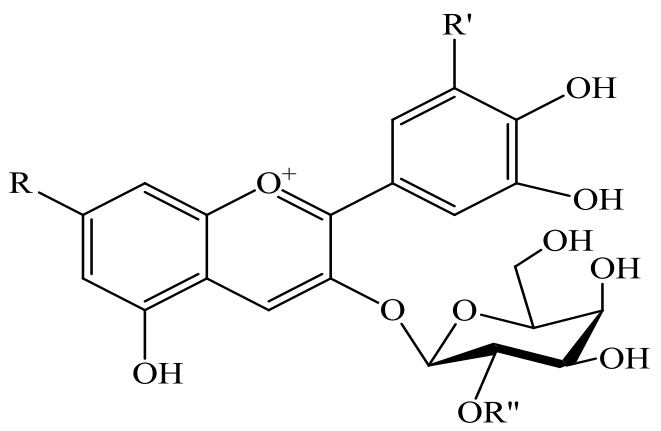<smiles>CC(=O)c1cc(O)c(O)c(O)c1</smiles>

R"=galloyl

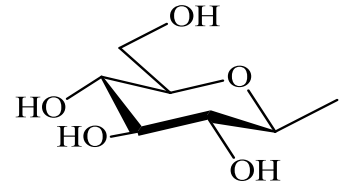

R"=glucosyl

$3 \mathrm{R}=\mathrm{OCH}_{3}, \mathrm{R}^{\prime}=\mathrm{H}, \mathrm{R}=$ galloyl

$4 \mathrm{R}=\mathrm{OCH}_{3}, \mathrm{R}^{\prime}=\mathrm{H}, \mathrm{R}^{\prime \prime}=\mathrm{H}$

$5 \mathrm{R}=\mathrm{OCH}_{3}, \mathrm{R}^{\prime}=\mathrm{OH}, \mathrm{R}^{\prime \prime}=$ galloyl

$6 \mathrm{R}=\mathrm{OH}, \mathrm{R}^{\prime}=\mathrm{H}, \mathrm{R} "=$ galloyl<smiles></smiles>

Figure 2

7

8

10

Effect of anti-inflammation of 1

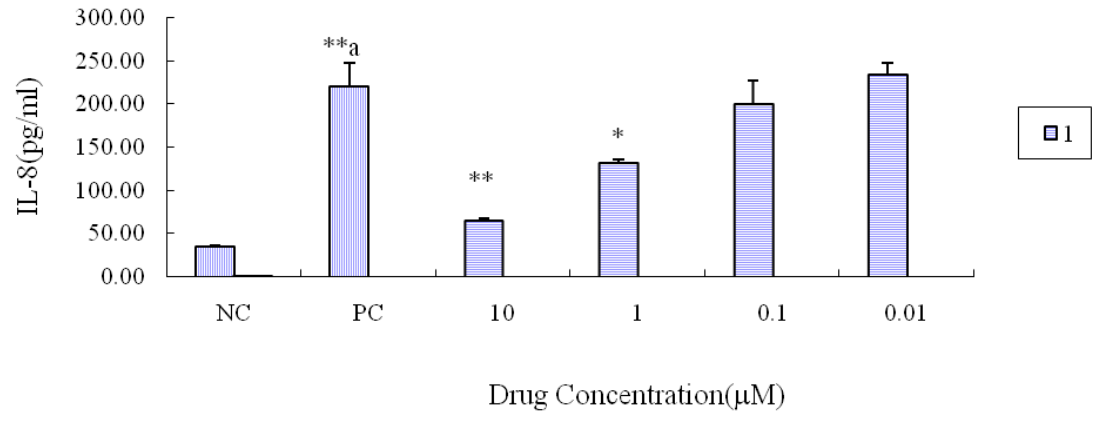



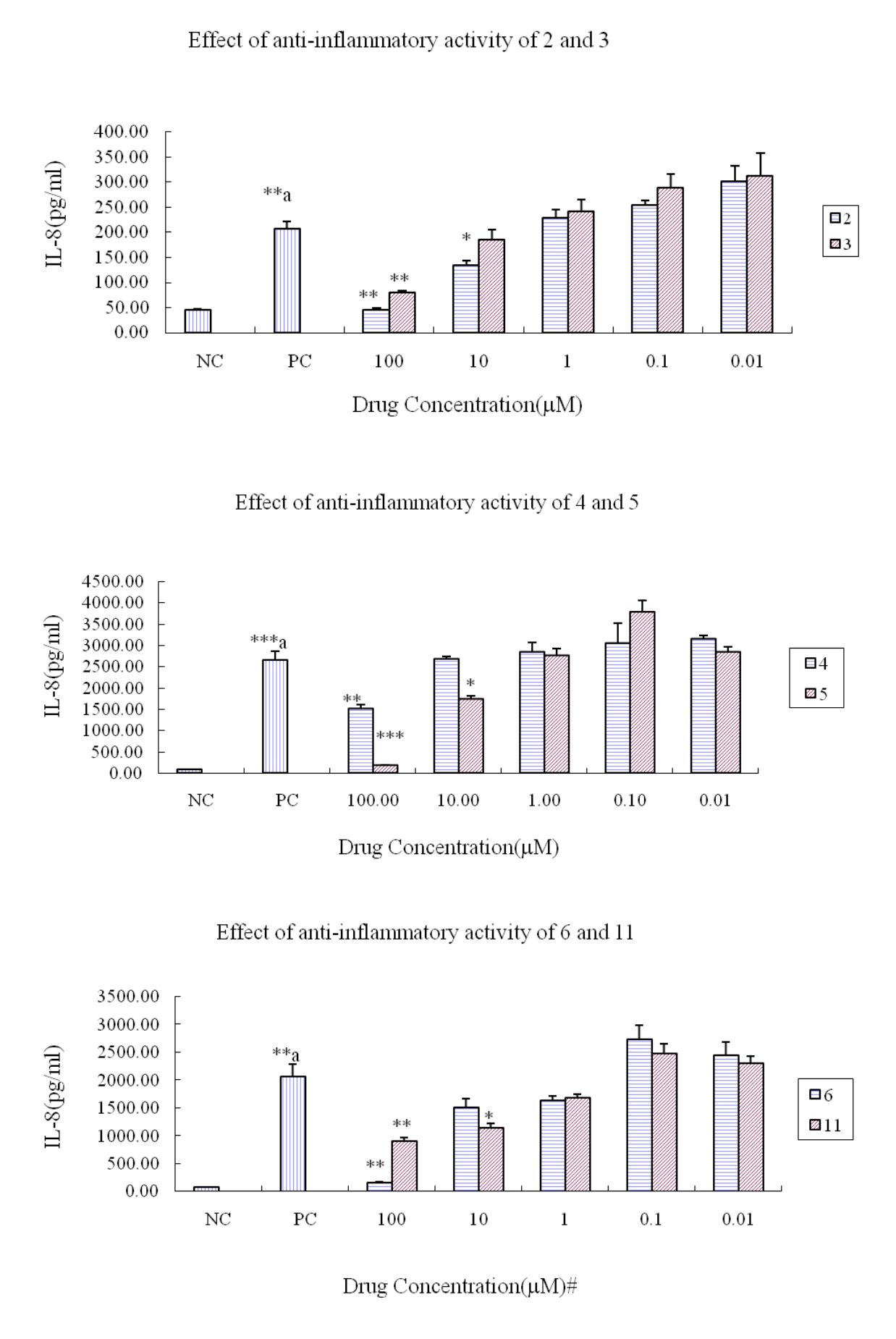


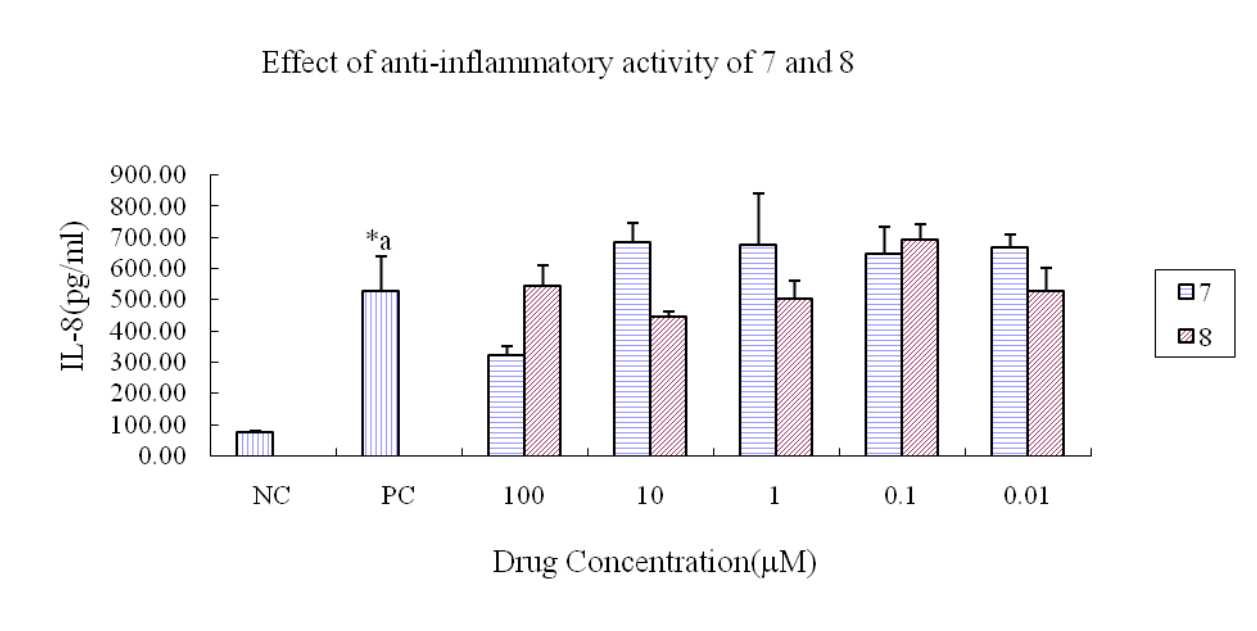

Effect of anti-inflammatory activity of 9 and 10

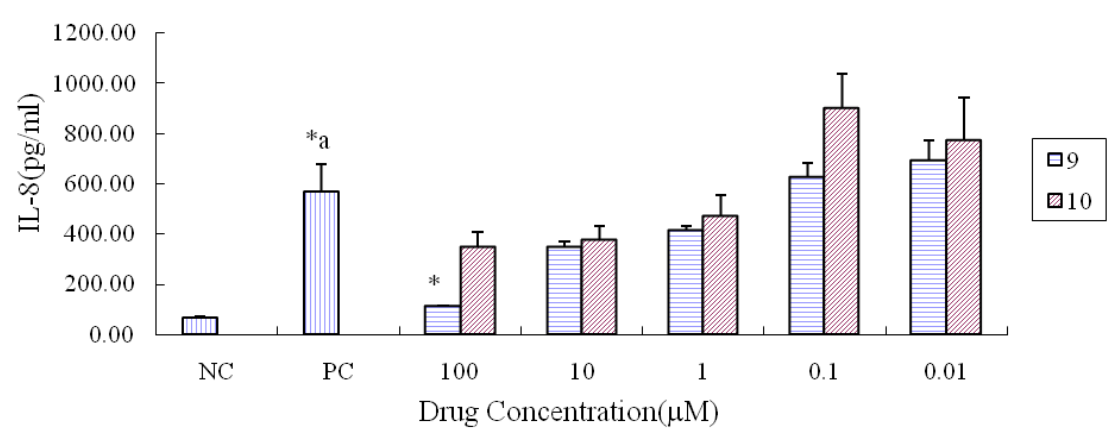

Figure 3

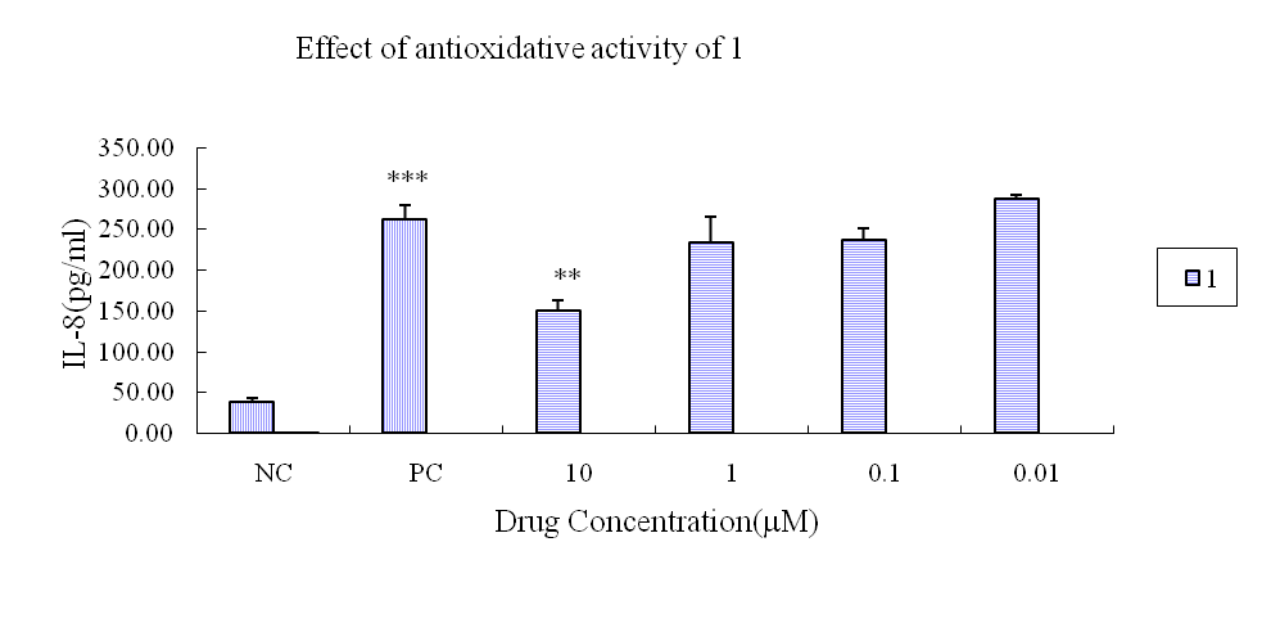



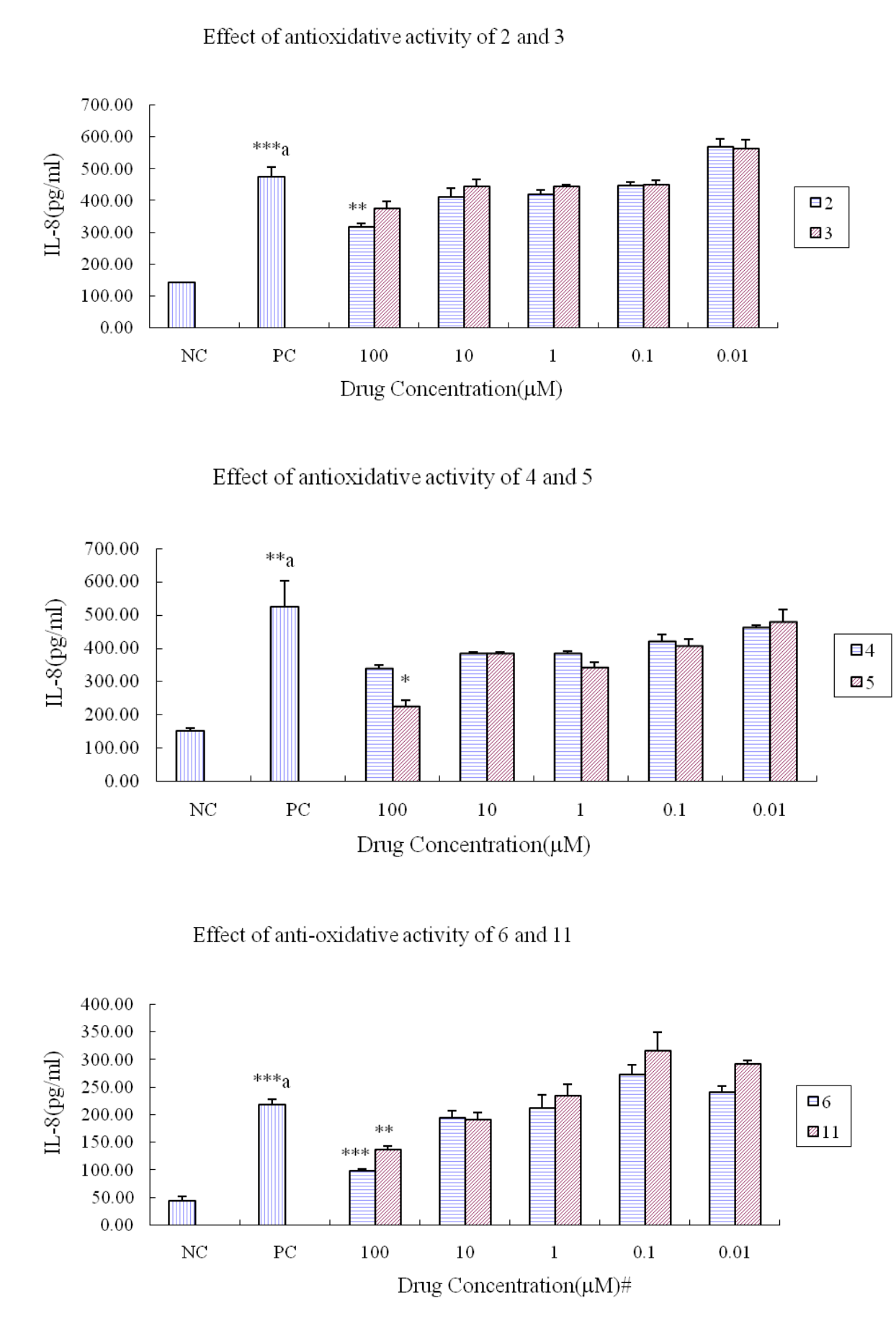
Effect of antioxidative activity of 7 and 8

$3364 \pm 640$

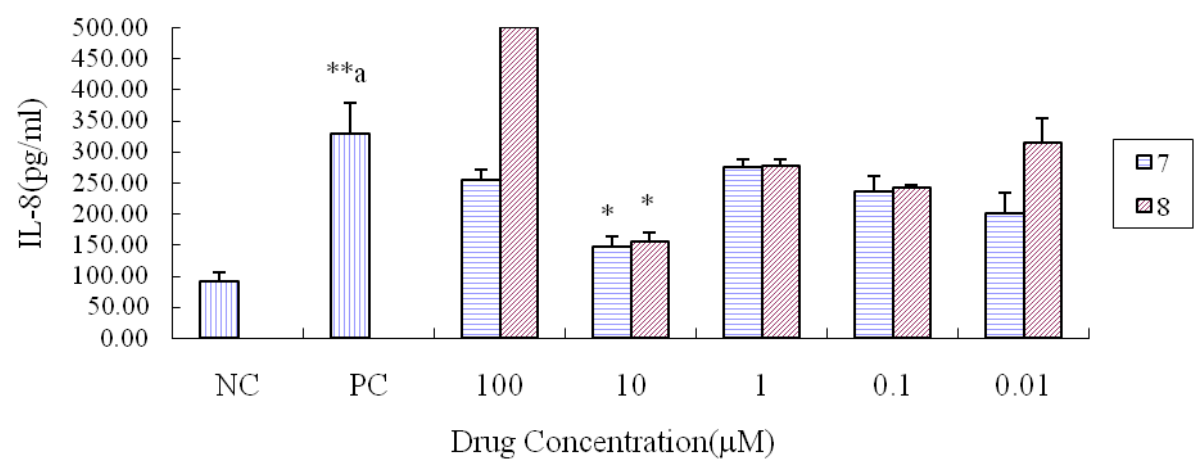

Effect of antioxidative activity of 9 and 10
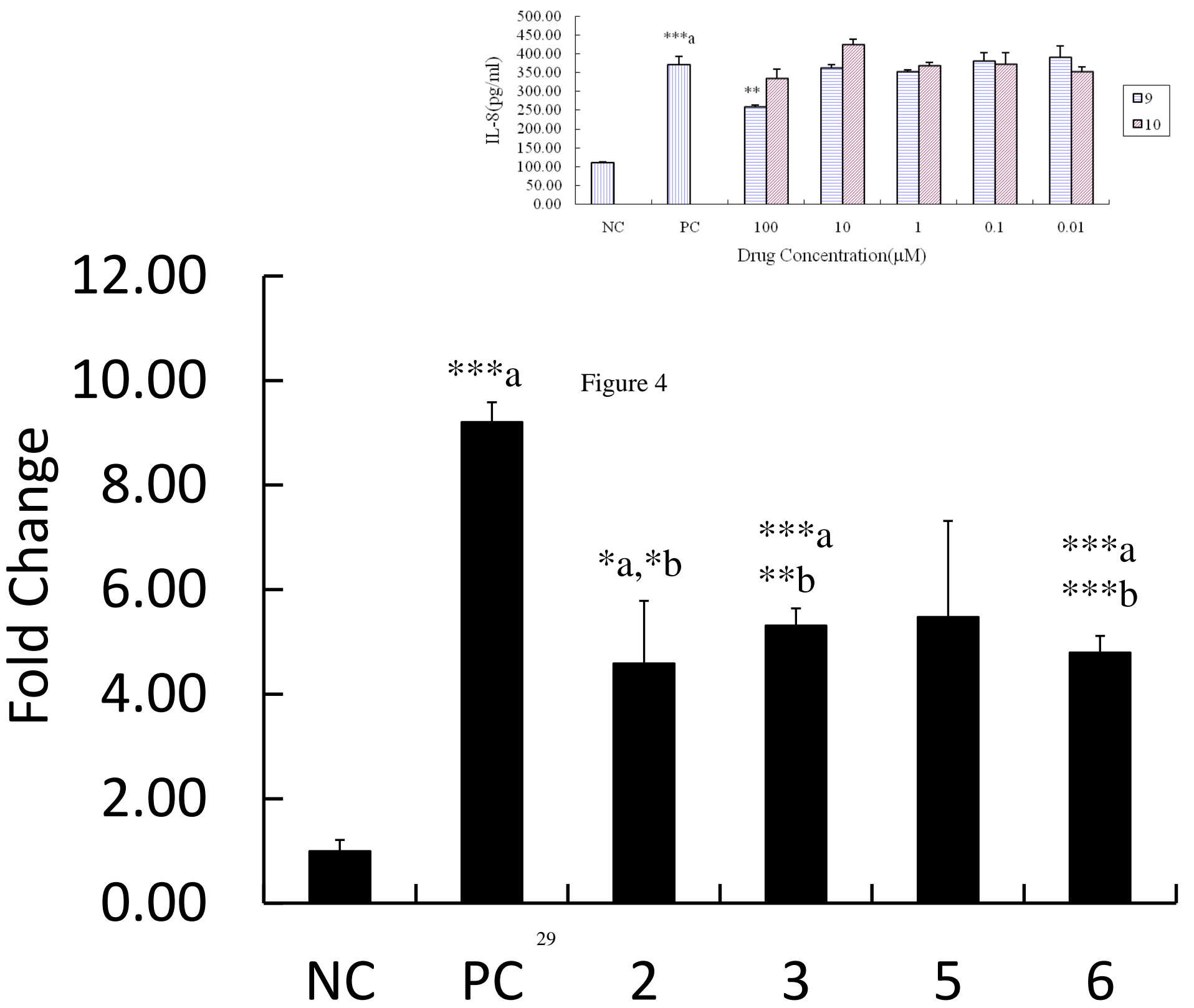
Table 1 Anti-inflammatory activity of Sumac polyphenols

\begin{tabular}{ccccccc}
\hline Compound & Compound & \multicolumn{5}{c}{ Concentration $(\mu \mathrm{M})^{\mathrm{a}}$} \\
\cline { 3 - 7 }$\#$ & Name & 100 & 10 & 1 & 0.1 & 0.01 \\
\hline $\mathbf{1}$ & & NT & $* *$ & $*$ & - & - \\
$\mathbf{2}$ & & $* *$ & $*$ & - & - & - \\
$\mathbf{3}$ & & $* *$ & - & - & - & - \\
$\mathbf{4}$ & $* *$ & - & - & - & - \\
$\mathbf{5}$ & $* * *$ & $*$ & - & - & - \\
$\mathbf{6}$ & $* *$ & - & - & - & - \\
$\mathbf{7}$ & - & - & - & - & - \\
$\mathbf{8}$ & - & - & - & - & - \\
$\mathbf{9}$ & & - & - & - & - & - \\
$\mathbf{1 0}$ & & - & - & - & - & - \\
$\mathbf{1 1}$ & & $* *$ & $*$ & - & - & - \\
\hline
\end{tabular}

NT, not tested, -, no significant difference $(\mathrm{P}>0.05), *, \mathrm{P}<0.05, * * \mathrm{P}<0.01$, and $* * * \mathrm{P}<0.001$ relative to the positive control (TNF- $\alpha$-treated cells). a: the concentration of 11 is $\mathrm{mg} / \mathrm{L}$. 
Table2 the antioxidative activity of Sumac polyphenols

\begin{tabular}{ccccccc}
\hline Compound & Compound & \multicolumn{5}{c}{ Concentration $(\mu \mathrm{M})^{\mathrm{a}}$} \\
\cline { 2 - 7 }$\#$ & Name & 100 & 10 & 1 & 0.1 & 0.01 \\
\hline $\mathbf{1}$ & $\mathrm{NT}$ & $* *$ & - & - & - \\
$\mathbf{2}$ & $* *$ & - & - & - & - \\
$\mathbf{3}$ & - & - & - & - & - \\
$\mathbf{4}$ & - & - & - & - & - \\
$\mathbf{5}$ & $*$ & - & - & - & - \\
$\mathbf{6}$ & $* *$ & - & - & - & - \\
$\mathbf{7}$ & $* *$ & $*$ & - & - & - \\
$\mathbf{8}$ & $* * \mathrm{~b}$ & $*$ & - & - & - \\
$\mathbf{9}$ & $* *$ & - & - & - & - \\
$\mathbf{1 0}$ & - & - & - & - & - \\
$\mathbf{1 1}$ & & $* *$ & - & - & - & - \\
\hline
\end{tabular}

NT: not tested, -: No significant difference, $* \mathrm{P}<0.05, * * \mathrm{P}<0.01$, and $* * * \mathrm{P}<0.001$ relative to positive control (TNF- $\alpha$ ) cells. a: the concentration of 11 is $\mathrm{mg} / \mathrm{L}$. **b, pro-oxidant 